\title{
CrystEngComm
}

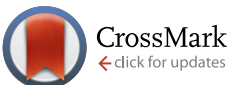

Cite this: CrystEngComm, 2014, 16, 11102

\section{Particle formation pathways and polymorphism of curcumin induced by ultrasound and additives during liquid antisolvent precipitation $\dagger$}

\author{
Alpana A. Thorat and Sameer V. Dalvi*
}

\begin{abstract}
Precipitation of curcumin, a poorly water-soluble drug, has been carried out by the liquid antisolvent technique in the presence of ultrasound and stabilizers. Curcumin particles with varied morphology were observed to have formed during precipitation in the presence of ultrasound and additives such as sodium dodecyl sulfate (SDS), Tween 80, hydroxyl propyl methylcellulose (HPMC), polyvinyl pyrrolidone (PVP), and bovine serum albumin (BSA). Characterization of the precipitated particles reveals that curcumin particles precipitated with ultrasound and large polymeric stabilizers such as HPMC, PVP, and BSA are superstructures formed by aggregation of several primary curcumin nanoparticles. The particles in these cases appeared to be loose aggregates (of $\sim 1-5 \mu \mathrm{m}$ in size) composed of several curcumin nanoparticles ( 50-200 $\mathrm{nm}$ in size). On the other hand, curcumin particles precipitated in the presence of ultrasound and without additive as well as small-molecule stabilizers like SDS and Tween 80 appear more fused (the primary building blocks of these particles were not clearly visible). Moreover, curcumin particles precipitated in the presence of ultrasound only and particles precipitated in the presence of ultrasound along with additives were found to exist in the orthorhombic form, whereas raw curcumin and curcumin particles precipitated without any additive and without ultrasound were found to exist in the monoclinic form. The results obtained in this work suggest that curcumin particles with the desired physical form and morphology can be engineered through a careful manipulation/choice of ultrasound and additives during precipitation.
\end{abstract}

Received 6th October 2014, Accepted 30th October 2014

DOI: $10.1039 / c 4 c e 02021 a$

www.rsc.org/crystengcomm

\section{Introduction}

Liquid antisolvent (LAS) precipitation is one of the bottomup techniques to produce nanoparticles of poorly watersoluble active pharmaceutical ingredients (APIs). ${ }^{1}$ Various drug formulation strategies have been extensively studied for such drugs over the past few decades to enhance their dissolution rates. ${ }^{2}$ One of the formulation strategies is to prepare nanoparticles/microparticles of these drugs. A decrease in particle size increases the surface-to-volume ratio and hence their rate of dissolution. ${ }^{2}$ The liquid antisolvent (LAS) process provides an attractive alternative to most of the available bottom-up methods in a way that it can be carried out

Chemical Engineering, Indian Institute of Technology Gandhinagar, Chandkheda, Ahmedabad-382424, Gujarat, India. E-mail: sameervd@iitgn.ac.in;

Fax: +091 792397 2324; Tel: +091 7932419529

$\dagger$ Electronic supplementary information (ESI) available: Experimental materials and methods, table on precipitated curcumin form details, comparative XRPD patterns of form 1 , form 2 and form 3 with the precipitated curcumin particles, hydrogen bonding in different curcumin forms, FTIR spectra of precipitated curcumin particles, and the SEM micrograph of raw curcumin. See DOI: 10.1039/c4ce02021a at ambient temperatures and atmospheric pressure with no requirement of expensive equipment (with easy scalability). ${ }^{1}$ In LAS precipitation, addition of a non-solvent to the solution of solute in solvent creates supersaturation resulting in the precipitation of solute. The precipitation of solute via LAS has been considered to follow a classical crystallization pathway where nuclei formed subsequent to the generation of supersaturation grow by the process of molecular addition. ${ }^{3-5}$ However, it has recently been shown that the LAS precipitation of organic compounds $\mathrm{s}^{6-12}$ also follows a non-classical pathway where colloidal nanosized intermediates assemble in the presence of additives/direction to form superstructures. ${ }^{13}$ These superstructures are intermediates to crystalline particles and the crystallographic fusion of the constituents of these particles leads to the formation of crystalline particles.

The formation of such superstructures has widely been reported for many biominerals and several inorganic materials. ${ }^{13}$ However, reports on the occurrence of such superstructures observed during precipitation of organic materials are rare. There are a few studies on the formation of superstructures for organic materials such as amino acids like diphenylalanine,${ }^{14}$ DL-alanine,${ }^{6-8}$ DL-glutamic acid,${ }^{11}$ and 
L-cystine $^{12}$ and poorly water soluble drugs such as diclofenac ${ }^{9}$ and sodium ibuprofen dihydrate ${ }^{12}$ that have been reported.

In this paper, we present an account of the formation of superstructures of curcumin nanoparticles, precipitated from its ethanolic solution in the presence of ultrasound and additives using water as an antisolvent (experimental details are presented in the ESI $\dagger$ ). Curcumin is a poorly water-soluble drug found in the herbal spice turmeric (Curcumin longa). It has potential antioxidant, anti-inflammatory, antitumor, anti-HIV, and antimicrobial properties. ${ }^{15}$ We show that the use of ultrasound and stabilizers during precipitation of curcumin particles induces morphological transformations. Curcumin exists in three forms of which form 1 is monoclinic and the other two forms (form 2 and form 3) are orthorhombic. ${ }^{16,17}$ While unprocessed curcumin and curcumin particles precipitated without ultrasound and without stabilizers were present in the monoclinic form (form 1), the curcumin particles precipitated in the presence of ultrasound and stabilizers were found to exist in the orthorhombic form (form 2 and form 3). Based on the characterization of the precipitated particles, we propose that the non-classical pathways of particle formation are followed during precipitation of curcumin particles in the presence of ultrasound and large polymeric additives such as BSA, HPMC and PVP, where the primary curcumin nanoparticles $(\sim 50-200 \mathrm{~nm}$ in size) aggregate to form secondary superstructures which are 1-5 $\mu \mathrm{m}$ in size.

\section{Results and discussion}

\subsection{Physical form of precipitated curcumin particles}

Fig. 1 presents powder X-ray diffraction (PXRD) patterns of unprocessed curcumin and precipitated curcumin particles, and of monoclinic (form 1) and orthorhombic forms (form 2 and form 3) of curcumin available in the literature. ${ }^{16,17}$ Comparison of PXRD patterns for unprocessed curcumin and curcumin particles precipitated without ultrasound and without stabilizer clearly indicates that these particles exist in form 1 (monoclinic form). On the other hand, curcumin particles precipitated in the presence of ultrasound only and the curcumin particles precipitated in the presence of ultrasound and stabilizers such as SDS, Tween 80, BSA, HPMC exist in form 3 (orthorhombic form). Similarly, curcumin particles precipitated in the presence of ultrasound and PVP were found to exist in form 2 (orthorhombic form) (see the ESI $\dagger$ Table S1 and Fig. S1 to S12 for form identification).

The monoclinic form (form 1) of curcumin differs from the orthorhombic form (forms 2 and 3) mainly in terms of the conformation of a curcumin molecule and its interaction through hydrogen bonding with the neighboring curcumin molecules. In the monoclinic form, curcumin molecules form a macrocyclic hydrogen bond ring due to phenol $\mathrm{O}-\mathrm{H} \cdots \mathrm{O}$ interactions among the curcumin molecules ${ }^{16}$ (Fig. S13†). Such a hydrogen bond ring is absent in the orthorhombic form (Fig. S14 and S15 $\dagger$ ). In the monoclinic form, the curcumin molecule has a curved and slightly twisted conformation, whereas it has a linear and planar conformation in the orthorhombic form ${ }^{16}$ (Fig. S16 $\dagger$ ). It could therefore be suggested that the precipitation of curcumin particles with the orthorhombic curcumin form (instead of the monoclinic form) in the presence of ultrasound could possibly be attributed to the prevention of hydrogen bonding interactions among curcumin molecules by ultrasound. Similar observations have been made during sonocrystallization of several APIs such as $p$-aminobenzoic acid, L-glutamic acid, sulfamerazine, and glycine, ${ }^{18-22}$ where the ultrasound was found to interrupt the intermolecular interactions

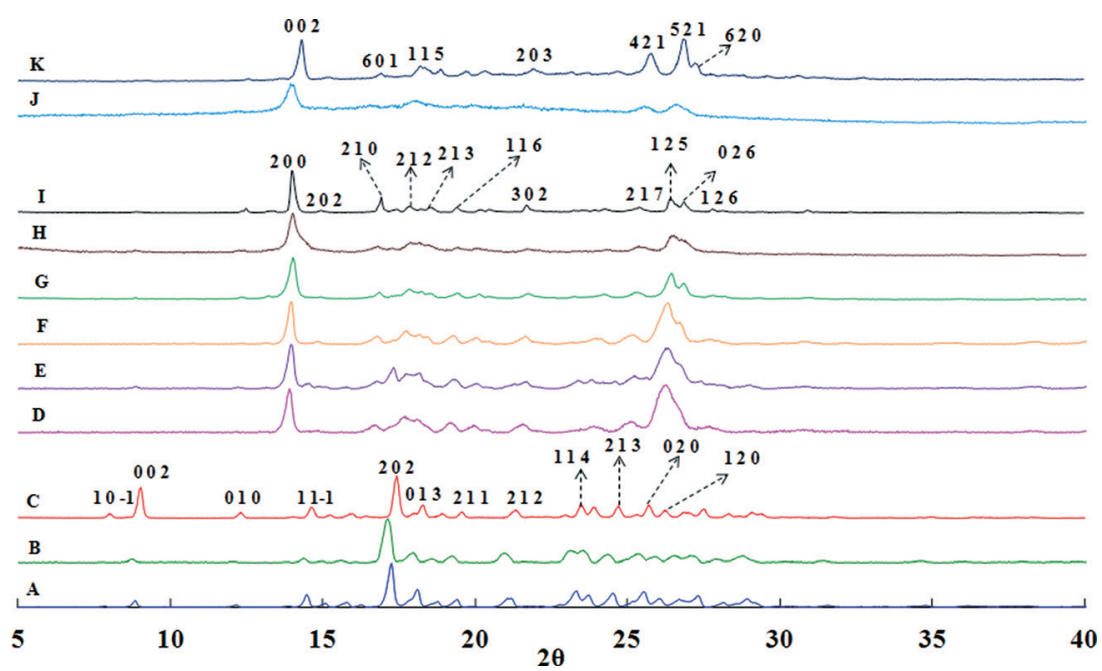

Fig. 1 Overlay of powder X-ray diffraction patterns of (A) Raw Curcumin, (B) Curcumin particles precipitated without ultrasound and without stabilizer, (C) Form 1 (monoclinic form) of Curcumin, ${ }^{16,17}$ and Curcumin particles precipitated in presence of (D) ultrasound and without stabilizer, (E) ultrasound and SDS, (F) ultrasound and Tween 80, (G) ultrasound and BSA, (H) ultrasound and HPMC, (I) Form 3 (orthorhombic form) of Curcumin $^{16,17}$, (J) ultrasound and PVP, (K) Form 2 (orthorhombic form) of Curcumin. ${ }^{16,17}$ 
during the stage of critical nuclei formation and bring about the polymorphic transformations.

\subsection{Morphology of curcumin superstructures formed in the presence of ultrasound and stabilizers}

Scanning electron microscopy (SEM) analysis of curcumin particles reveals interesting morphological characteristics. The morphology of curcumin particles varies depending upon the variation in the presence or absence of ultrasound and the type of stabilizer used. Fig. 2a presents SEM images of curcumin particles precipitated without ultrasound and without stabilizers. It is interesting to observe that particles in this case exhibit a flower-like morphology at $0 \mathrm{~h}$ which are typically $\sim 4-5 \mu \mathrm{m}$ in size. Fig. $2 \mathrm{~b}$ presents the SEM image of these particles after $24 \mathrm{~h}$ from the time of precipitation, where dendritic structures could be observed. Each individual particle (around 35-40 $\mu \mathrm{m}$ in length) consists of a stem-like middle portion (which is $\sim 5 \mu \mathrm{m}$ in size, shown in Fig. 2c) from where the extended tentacles (each of $\sim 15-17 \mu \mathrm{m}$ in size) originate. It is interesting to observe from Fig. $2 \mathrm{a}$ that the particles at $0 \mathrm{~h}$ show ends with small finger-like protrusions, which indicates an onset of particle branching. ${ }^{23}$ The finger-like protrusions later grow into large tentacles at $24 \mathrm{~h}$ (Fig. 2b).

Fig. 2(d-f) present SEM micrographs of curcumin particles precipitated in the presence of $105 \mathrm{~W}$ ultrasound but no stabilizer. It can be observed that particles at $0 \mathrm{~h}$ exhibit a long rice seed-like morphology with the length of the particles around $\sim 5 \mu \mathrm{m}$ (Fig. 2d). The particles appear to consist of several layers of curcumin with prominent growth along the $c$-axis. These particles at $0 \mathrm{~h}$ show signs of initial sprouting of tentacles but the tentacles do not grow at $24 \mathrm{~h}$ (Fig. 2e) unlike the particles precipitated in the absence of ultrasound (Fig. 2b). In order to understand the process of particle formation, a time-resolved SEM analysis was carried out for precipitation of curcumin in the presence of ultrasound only (no stabilizer). In these studies, the SEM images were recorded for the samples taken out from the solution immediately after addition of antisolvent ( $>5 \mathrm{~s}$ ) and then at 1, 3, and 10 minutes during sonication. These SEM images are presented in Fig. 3. It can be observed clearly that at 0 minute, the solution contained a large number of curcumin particles of nearly 50-100 nm size (Fig. 3a-b) and some bigger particles which are formed by the aggregation of the primary nanoparticles by end-to-end attachment. Such particles could be observed in the sample taken at 1 minute as well (Fig. $3 \mathrm{c}-\mathrm{d}$ ). After 3 minutes from the addition of antisolvent, the end-to-end attachment of primary nanoparticles in single particles was still visible and particles did not look completely fused (Fig. 3e-f). By the end of 10 minutes, however, the particles looked more fused and layers were less visible (Fig. $3 g-h$ ). Thus, a time-resolved SEM analysis clearly indicated that curcumin particles precipitated without stabilizer and in the presence of ultrasound are made up of layers formed by the end-to-end attachment of primary curcumin nanoparticles.

SEM micrographs of curcumin particles precipitated in the presence of ultrasound and with SDS are shown in Fig. $2(\mathrm{~g}-\mathrm{i})$. These particles (which are $\sim 3 \mu \mathrm{m}$ in size) possess a rice-seed like morphology with truncated ends. Curcumin particles precipitated with ultrasound and Tween 80 as a stabilizer shown in Fig. $2(\mathrm{j}-\mathrm{l})$ are $\sim 1-2 \mu \mathrm{m}$ in size and possess a mixed morphology consisting of rice-seed-like particles with truncated ends as well as flat plate-like particles. In both these cases, particles show layers of curcumin fused together to form a complete particle. The concentration of the stabilizer ( $0.02 \mathrm{wt} \%$ of the aqueous suspension) used turns out to be below that of CMC in the case of SDS and above that of CMC in the case of Tween 80. To understand the process of particle formation in these cases, timeresolved SEM analyses were carried out for curcumin particles precipitated in the presence of ultrasound and with SDS and Tween 80 as well (Fig. 4 and 5). In the case of curcumin particles precipitated in the presence of ultrasound and SDS, the particles at 0 minute appear to have been made of layers of curcumin (Fig. 4a and b). The particles, however, seem to have undergone fusion later as evident from the SEM image of a sample taken at 3 minutes (Fig. $4 \mathrm{e}$ and $\mathrm{f}$ ) and 10 minutes (Fig. $4 \mathrm{~g}$ and $\mathrm{h}$ ). At 10 minutes, breakage of curcumin particles due to ultrasound also seems to have occurred (Fig. $4 \mathrm{~g}$ and $\mathrm{h}$ ). In the case of curcumin particles precipitated with ultrasound and Tween 80 , the primary building blocks of the particles were not very visible in any of the time-resolved SEM images (Fig. 5).

While curcumin particles precipitated in the presence of ultrasound and small-molecule stabilizers like SDS and Tween 80 mainly exhibit rice-seed like (fused) morphology, curcumin particles precipitated with polymeric stabilizers such as BSA, HPMC, and PVP clearly look like aggregates formed by the assembly of several nanoparticles. The particles precipitated in the presence of BSA and ultrasound appear to be spherical aggregates $\sim 1 \mu \mathrm{m}$ in size made from aggregation of several primary nanoparticles [shown in Fig. 2(m-o)]. These primary nanoparticles were found to be plate-like as well as spherical with a size of $\sim 100-200 \mathrm{~nm}$ (see Fig. 2o). In the presence of ultrasound and HPMC, rice seed-like superparticles of $\sim 2-5 \mu \mathrm{m}$ were obtained [shown in Fig. 2(p-r)]. The primary constituents of these particles were found to be small rod-shaped nanoparticles around $100 \mathrm{~nm}$ in size (see Fig. 2r). Precipitation of curcumin in the presence of PVP and ultrasound [shown in Fig. 2(s-u)] results in spherical pearl-like aggregates $\sim 1.5 \mu \mathrm{m}$ in size, where the primary units were spherical nanoparticles with a size of $\sim 50-80 \mathrm{~nm}$ (see Fig. $2 \mathrm{u}$ ).

The AFM analysis of the precipitated particles yielded additional evidence of the aggregation of curcumin nanoparticles into superstructures (Fig. 6). It can be clearly observed from the AFM image of the curcumin particle precipitated in the presence of ultrasound and without stabilizer (Fig. 6A) that this particle is made up of subunits 

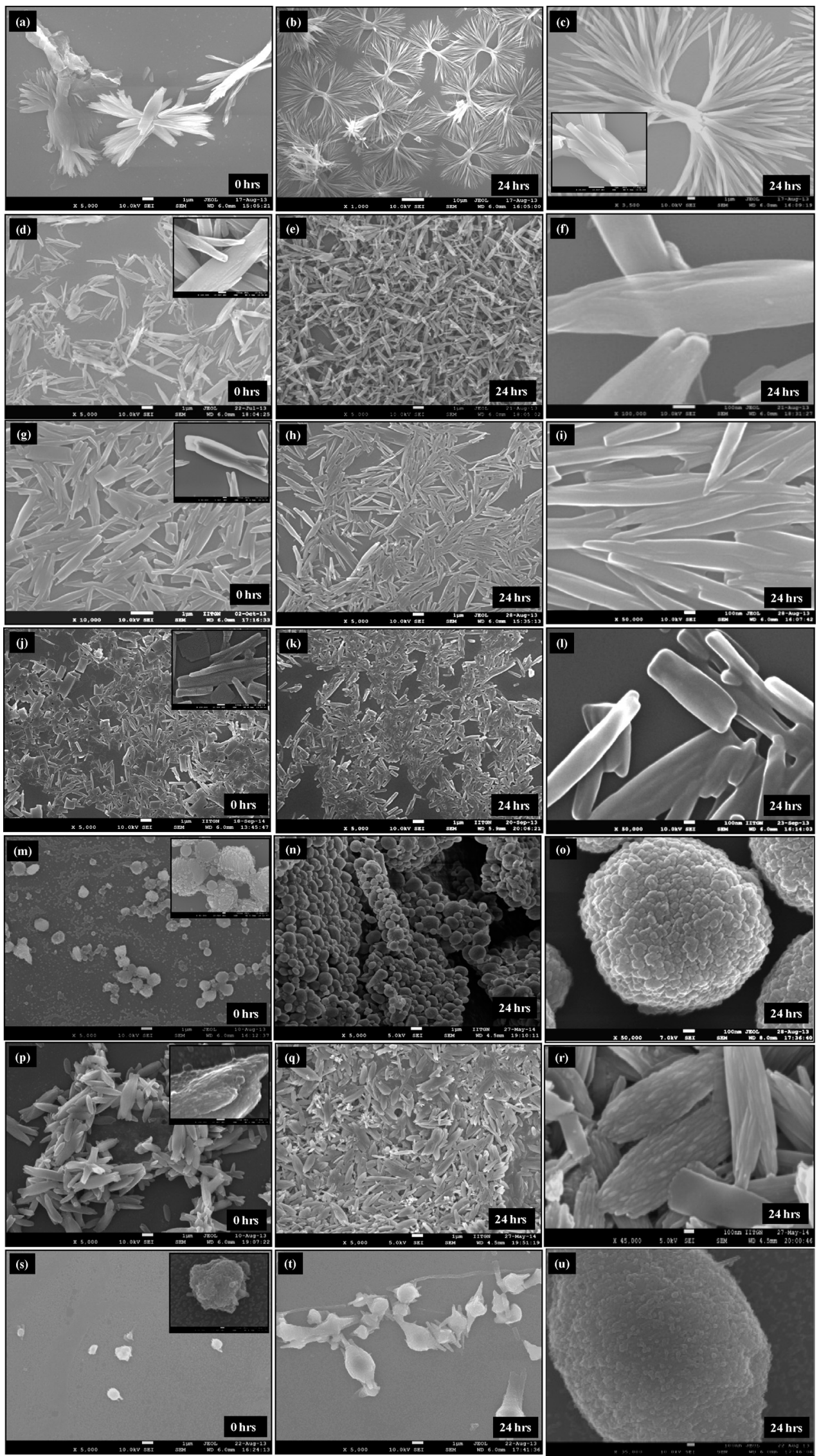

Fig. 2 SEM micrographs of curcumin particles precipitated without ultrasound and without stabilizer (a-c), with $105 \mathrm{~W}$ ultrasound and (d-f) without surfactant, $(\mathrm{g}-\mathrm{i})$ with SDS, $(\mathrm{j}-\mathrm{l})$ with Tween $80,(\mathrm{~m}-\mathrm{o})$ with BSA, $(\mathrm{p}-\mathrm{r})$ with HPMC, and $(\mathrm{s}-\mathrm{u})$ with PVP. 

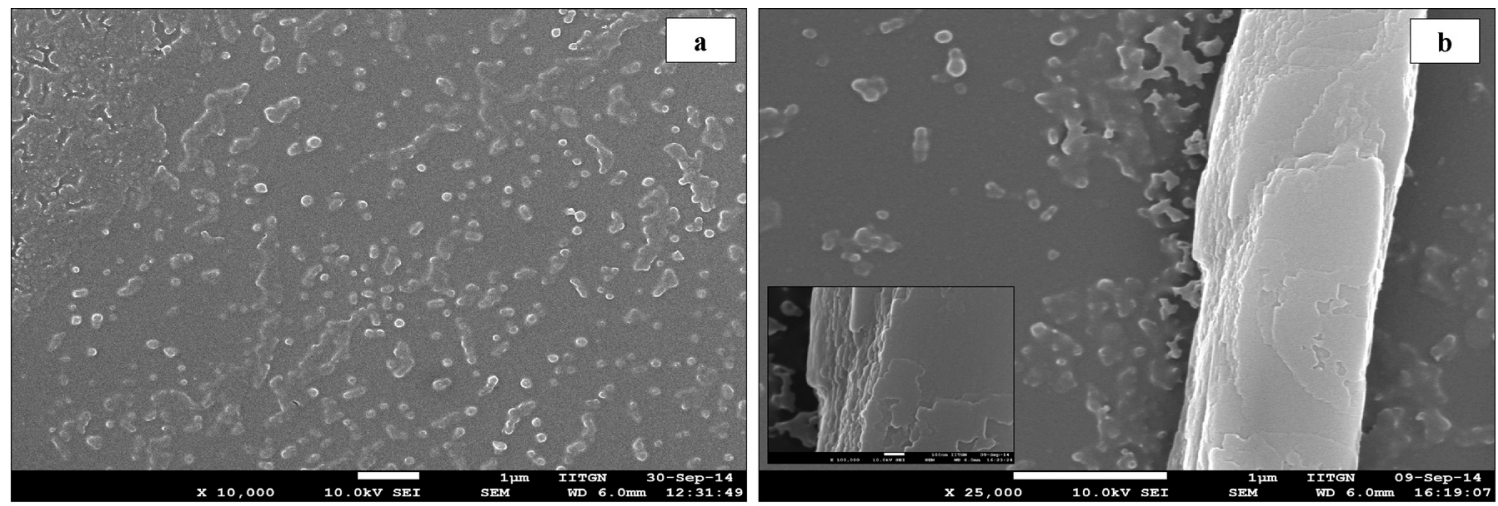

$0 \mathrm{~min}$
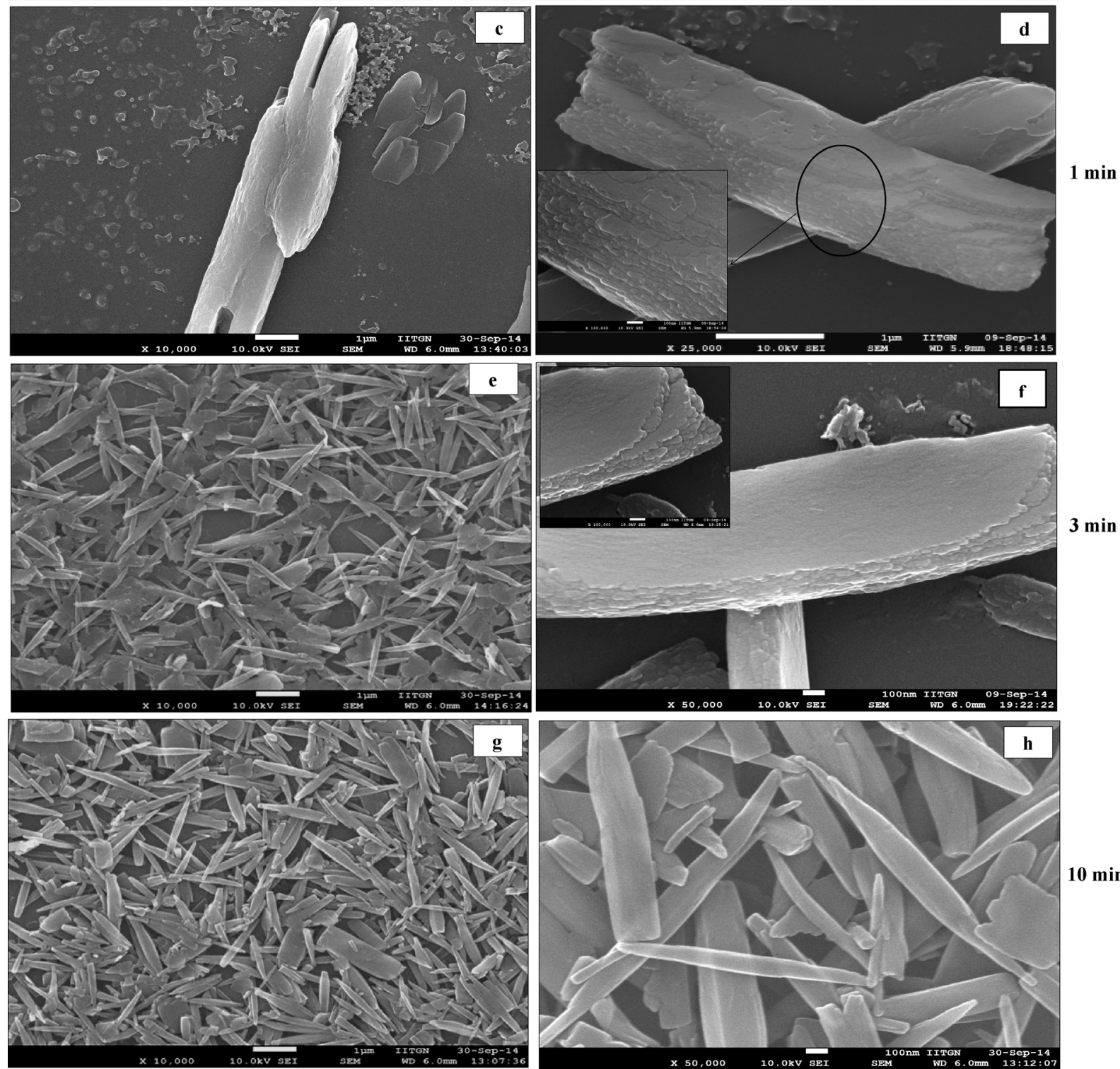

3 min

$10 \mathrm{~min}$

Fig. 3 Time-resolved SEM micrographs of samples taken out during sonication of curcumin particles precipitated with ultrasound and without stabilizer (a-b) at $0 \mathrm{~min},(\mathrm{c}-\mathrm{d})$ at $1 \mathrm{~min},(\mathrm{e}-\mathrm{f})$ at $3 \mathrm{~min}$, and (g-h) at $10 \mathrm{~min}$. The sequence of formation of building units and self-assembly in superstructure and subsequent fusion occurring during sonication is evident.

which are attached to each other possibly through the (001) plane and arranged in the $c$ direction (also confirmed by time-resolved SEM imaging shown in Fig. 3). The AFM image of the particles precipitated in the presence of SDS and ultrasound (Fig. 6B) and the AFM image of curcumin particles precipitated in the presence of Tween 80 



Fig. 4 Time resolved SEM micrographs of samples taken (a-b) at $0 \mathrm{~min}$, (c-d) at $1 \mathrm{~min}$, (e-f) at $3 \mathrm{~min}$, (g-h) at $10 \mathrm{~min}$, during precipitation of curcumin particles in presence of ultrasound and SDS.

and ultrasound (Fig. 6C), however, did not clearly present evidence of aggregation of primary nanoparticles. Instead, these images showed particles with rough surfaces. The
AFM image of curcumin particles precipitated with ultrasound and BSA shows randomly arranged subunits (Fig. 6D). The AFM image of the curcumin particle 

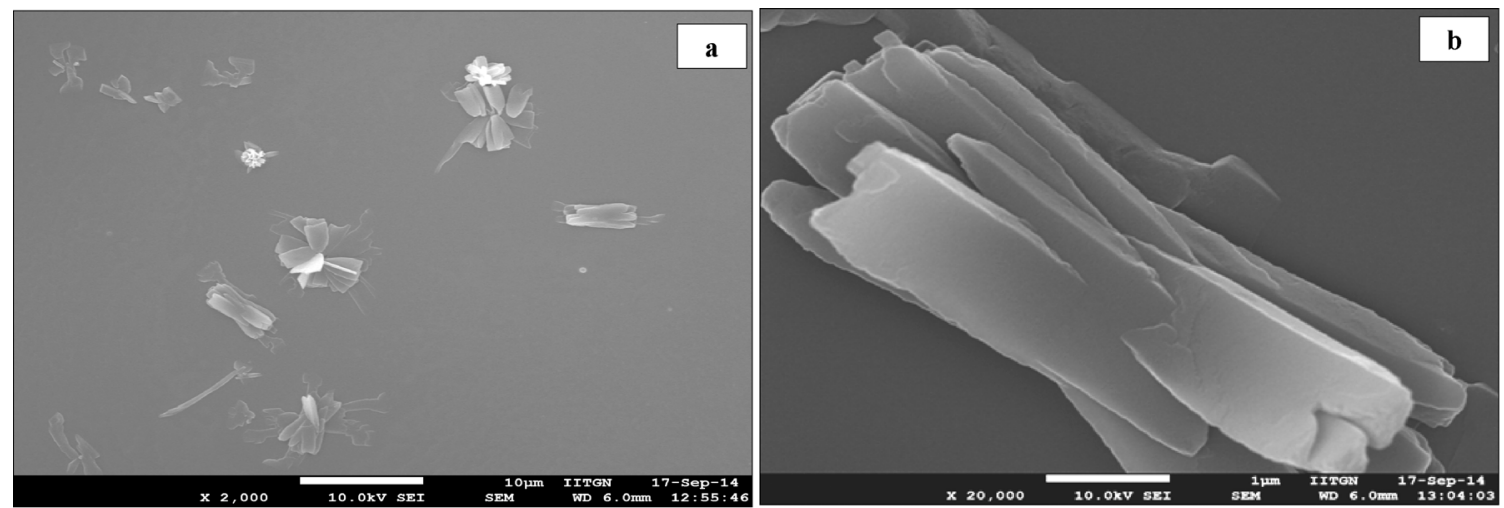

$0 \mathrm{~min}$
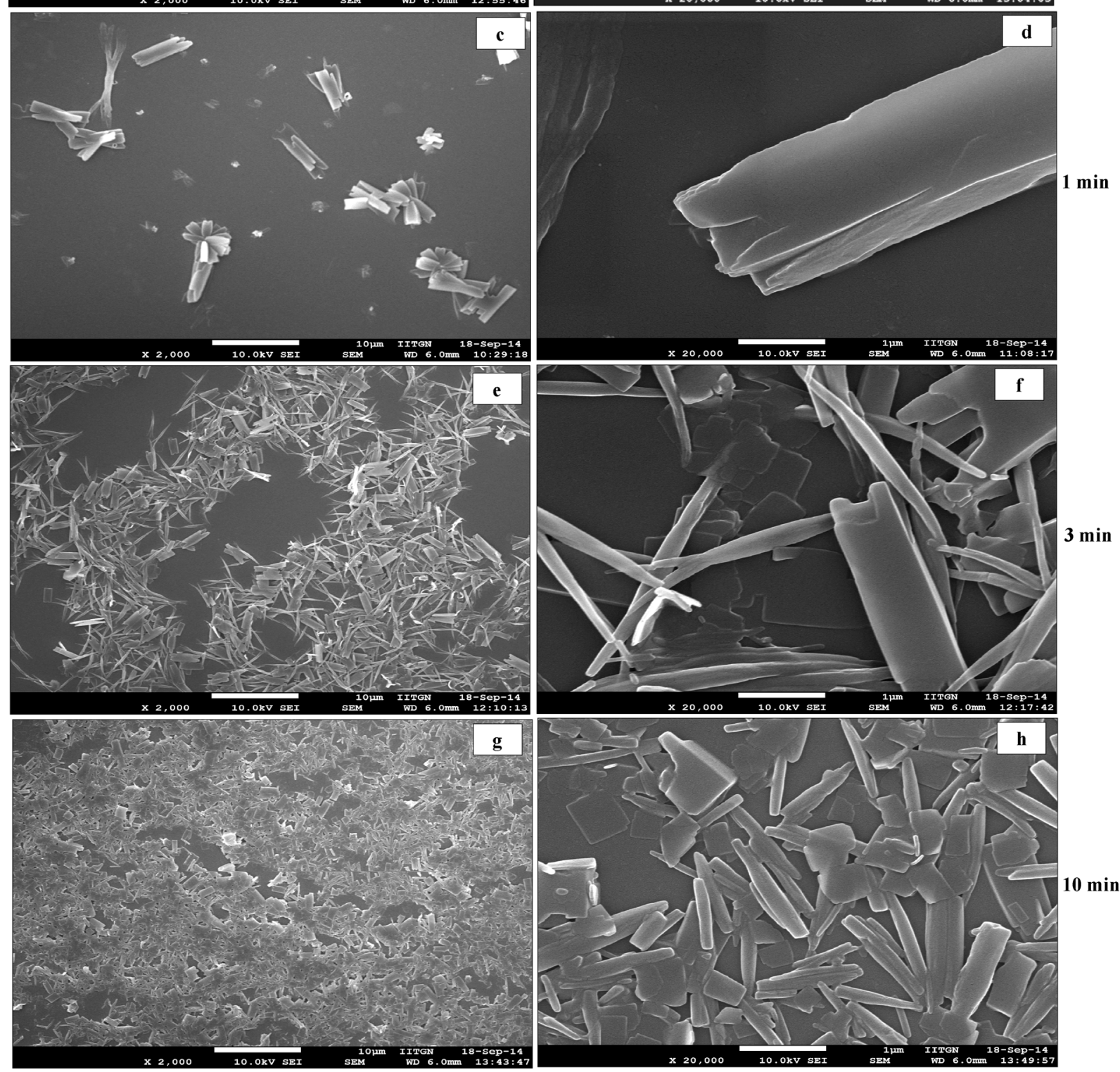

Fig. 5 Time resolved SEM micrographs of samples taken (a-b) at $0 \mathrm{~min},(\mathrm{c}-\mathrm{d})$ at $1 \mathrm{~min},(\mathrm{e}-\mathrm{f})$ at $3 \mathrm{~min},(\mathrm{~g}-\mathrm{h})$ at $10 \mathrm{~min}$, during precipitation of curcumin particles in presence of ultrasound and Tween 80 .

precipitated with ultrasound and HPMC shows primary nanoparticles arranged in the $c$-direction (Fig. 6E). Further, the curcumin particles precipitated with ultrasound and
PVP are randomly arranged (Fig. 6F). Although the exact arrangement of subunits in the particle is not completely visible with the help of AFM analysis, it still provides useful 

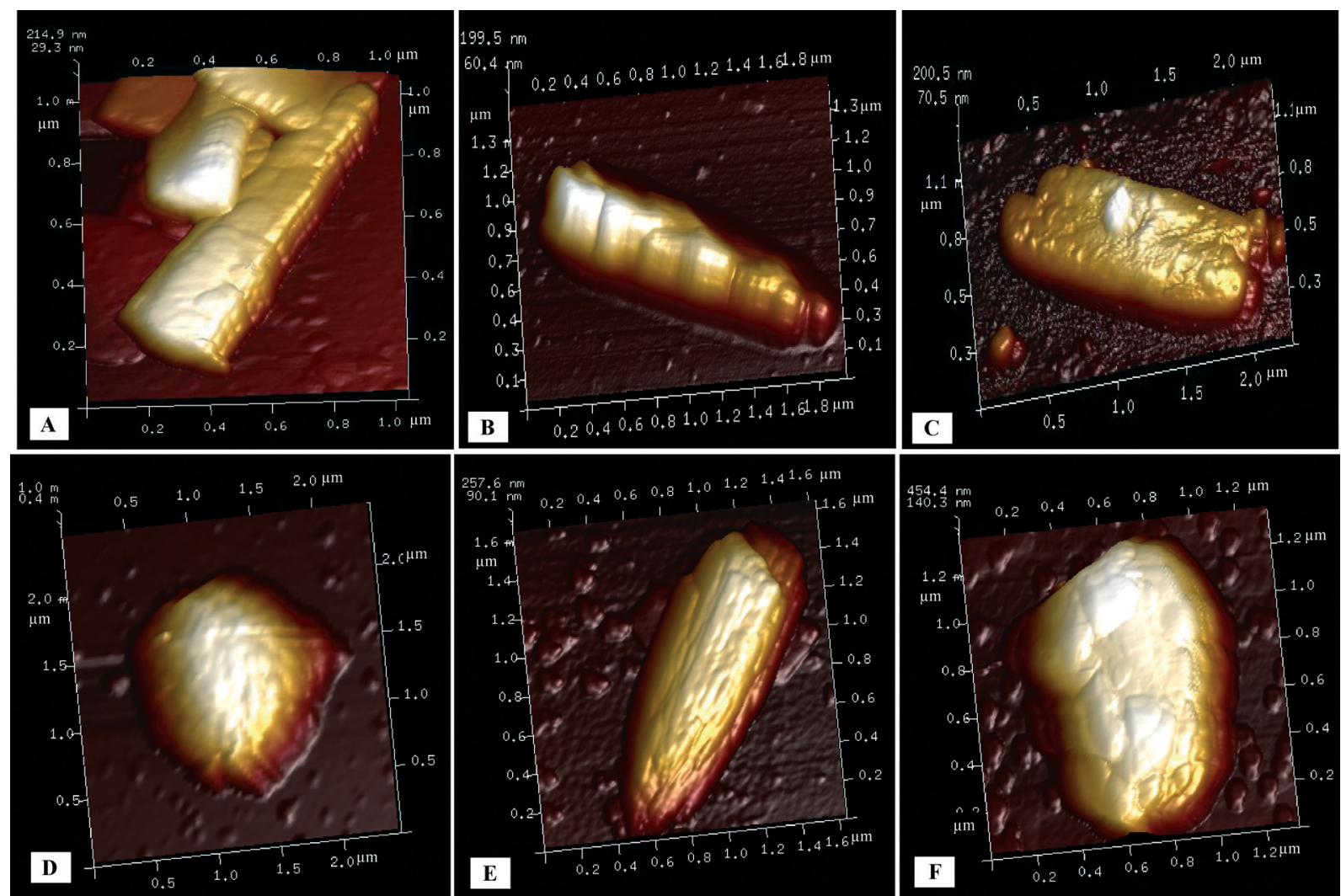

Fig. 6 AFM images of curcumin particles precipitated in the presence of ultrasound and (A) without stabilizer, (B) with SDS, (C) with Tween 80, (D) with BSA, (E) with HPMC, and (F) with PVP.

information on the self-assemblies of the small subunits into final superparticles.

In order to obtain further insight into the particle formation mechanisms, HR-TEM analysis of particles precipitated in the presence of ultrasound and stabilizers was carried out (Fig. 7). It can be observed from Fig. 7B that the SAED pattern of the curcumin particle precipitated in the presence of ultrasound and without stabilizer is a combination of rings as well as a few bright and discrete spots, indicating the existence of some crystallographic order among the primary constituents as well as the polycrystalline nature of these particles. On the other hand, a sharp ring-like pattern obtained from the particles precipitated in the presence of ultrasound and SDS indicates a strong polycrystalline nature of these particles (Fig. 7D). The SAED pattern of curcumin particles precipitated in the presence of ultrasound and Tween 80 (Fig. 7F) shows a diffuse zone with well-spread bright spots. Similarly, the SAED pattern of the curcumin particle precipitated in the presence of ultrasound and with BSA (Fig. 7H) shows sharp rings indicating the polycrystalline nature of these particles. The particles precipitated in the presence of ultrasound and HPMC, on the other hand, yield a diffraction pattern consisting of discrete as well as randomly spread spots indicating a mix of ordered as well as polycrystalline nature of these particles (Fig. 7J). However, in the case of curcumin particles precipitated in the presence of ultrasound and PVP, the SAED pattern (Fig. 7L) shows a diffuse zone with a few bright spots, indicating that these particles mainly consist of a matrix of amorphous subunits (also evident from XRD). Thus, SAED patterns of curcumin particles precipitated with stabilizers show traces of crystallinity to a varying extent. The extent of crystallinity is different in each case. In fact, in the case of curcumin particles precipitated with PVP and ultrasound, the precipitated particles were found to be mainly amorphous in nature.

\subsection{Proposed mechanism for formation of curcumin superstructures}

Based on the results of the characterization of precipitated particles, we can now propose hypothesized mechanisms for the formation of superstructures of curcumin with different morphologies.

The dendritic structures obtained by precipitation in the absence of ultrasound and no stabilizers are the result of the diffusion-limited growth mechanism ${ }^{24,25}$ (due to the limitation of mass transport in the absence of ultrasound). The absence of perfect mixing (no micromixing in the absence of ultrasound ${ }^{3,26,27}$ ) results in non-uniform zones of high supersaturation in the solution, which lead to uncontrolled growth of the particles (Fig. 8, path A). Such dendritic structures were not observed when ultrasound was used, where particle morphology was rice seed-like at $0 \mathrm{~h}$ as well as $24 \mathrm{~h}$. These observations possibly indicate an increased 



(F)
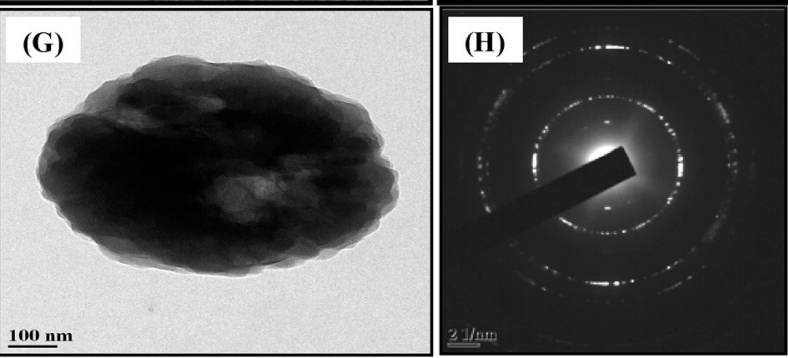

(I)
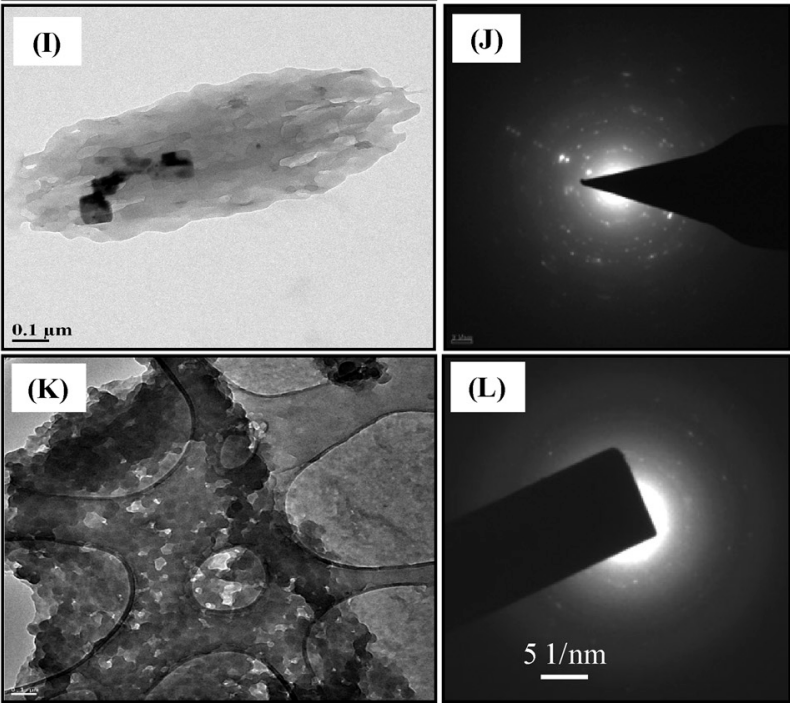

(L)

Fig. 7 TEM micrographs and SAED patterns of curcumin particles precipitated (A and B) with ultrasound and without stabilizer, (C and D) with ultrasound and with SDS, (E and F) with ultrasound and with Tween 80, (G and $\mathrm{H}$ ) with ultrasound and with BSA, (I and J) with ultrasound and with HPMC, and (K and $\mathrm{L}$ ) with ultrasound and with PVP. stability of curcumin particles precipitated in the presence of ultrasound against growth and the change in growth mechanism from diffusion-limited (due to the limitation of mass transport) in the absence of ultrasound to integration controlled (due to a well-mixed environment) in the presence of ultrasound (Fig. 8, path B). The change in growth mechanism could be attributed to the enhanced diffusivity of curcumin in aqueous solution under the influence of ultrasound. It has been reported in the literature that ultrasound increases the liquid-phase diffusivity of molecules by manifold. ${ }^{3,26,27}$ In our earlier study on liquid antisolvent precipitation of curcumin, it was found that $105 \mathrm{~W}$ ultrasound increases the diffusivity of curcumin in aqueous ethanolic solution by $300-400$-fold. ${ }^{28}$ In addition to the tremendous increase in diffusivity, ultrasound also enhances micromixing. ${ }^{3,26,27}$ These two effects together generate uniform supersaturation in the solution, which further helps in transforming localized and high-concentration gradients surrounding the growing particle surface (in the absence of ultrasound) into uniform and (hence) low-concentration gradients surrounding a growing crystal face (Fig. 8, path B). The suppression/lowering of the effective concentration gradients surrounding the growing crystal faces (or effective surface supersaturation) induces integration-controlled growth of particles.

Precipitation of curcumin particles with rice seed-like morphology found in the case of particles precipitated with ultrasound but no stabilizer can be explained with the help of the arrangement of curcumin molecules in the orthorhombic unit cell. Fig. 9 shows a typical orthorhombic unit cell (form 3) for curcumin with an arrangement of curcumin molecules in it. It is evident that the phenolic hydroxyl and phenolic methyl groups protrude out of the (001) plane (same groups in the case of orthorhombic form 2 are present on the (100) plane). These groups interact through hydrogen bonds with the phenolic methyl groups and phenolic hydroxyl groups of other curcumin molecules. Such interactions result in a growth by attachment of primary units along the $c$ axis, and hence the elongated rice seed-like particle morphology was observed in the case of particles precipitated with ultrasound but no stabilizer.

The use of stabilizers along with ultrasound, however, alters the particle morphology as explained in section 2.2, which is a result of curcumin-stabilizer interactions. The possible mechanisms for formation of particles with specific morphology due to stabilizer and curcumin interactions schematically presented in Fig. 10 is explained below. FTIR studies were carried out to investigate the interaction among curcumin and stabilizer molecules. FTIR spectra of curcumin particles precipitated in the presence of SDS along with ultrasound and in the presence of Tween 80 along with ultrasound show peak broadening at $3417 \mathrm{~cm}^{-1}$ and $3276 \mathrm{~cm}^{-1}$ positions when compared to FTIR spectra of curcumin particles precipitated with ultrasound and no stabilizer (Fig. S17 $\dagger$ ), indicating increased interaction of stabilizer molecules of SDS and Tween 80 with curcumin molecules. 


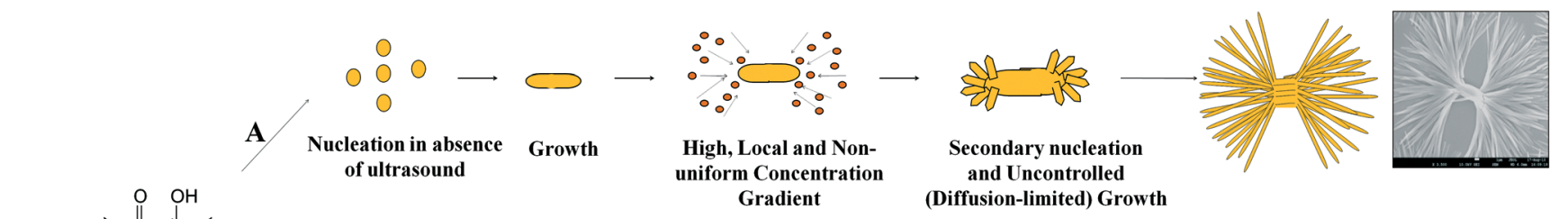

With Ultrasound only (no stabilizer)
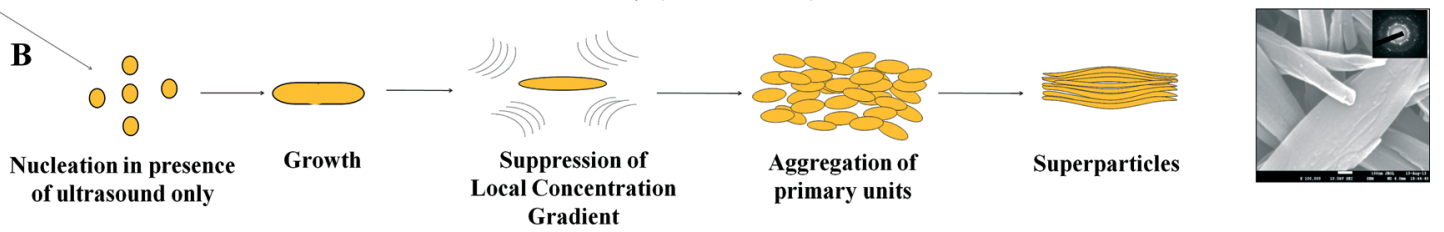

Symbols

Nuclei of Curcumin

Primary nanoparticles of curcumin

Curcumin molecule

Ultrasound waves

Fig. 8 Hypothesized mechanism of formation of curcumin superparticles in the absence and presence of ultrasound without stabilizers.

However, based on the evidence gathered from SEM, TEM, AFM and FTIR studies, it is difficult to propose an exact mechanism of particle formation in the presence of Tween 80 and SDS along with ultrasound. The observation of the morphology of these particles by SEM analysis (Fig. 2g-l) and also by time-resolved SEM studies (Fig. 4 and 5) does not clearly show primary building blocks or aggregates of primary nanoparticles. The particles in these cases look more fused. This possibly indicates that either a faster fusion of primary units into single particles has occurred or particle growth by the process of molecule-by-molecule addition has occurred. The exact mechanism is quite unclear. As mentioned earlier, the concentration of SDS and Tween $80(0.02 \mathrm{wt} \%$ of the aqueous solution) used turns out to be lower than that of CMC for SDS and higher than that of CMC for Tween 80. It has been reported in the literature that surfactants like SDS (at below CMC concentration) can act as growth inhibitors by adsorption on the particle surface. ${ }^{29}$ However, it is also possible that these surfactants may act as secondary nucleation

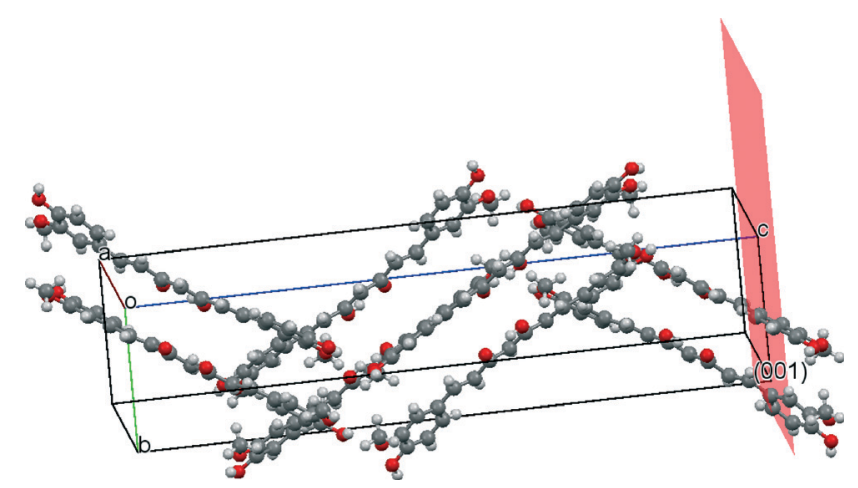

Fig. 9 Snapshot of molecular groups present on the $\left(\begin{array}{llll}0 & 0 & 1\end{array}\right)$ crystal plane of the orthorhombic curcumin form visualized using Mercury software version 3.1 . agents and cause growth of particles at below CMC levels. ${ }^{30}$ At concentrations above CMC, growth of particles can be accelerated by surfactant micelles by the mechanism of solubilisation of drug molecules into the micelles and transportation of these molecules to the particle surface. ${ }^{31}$ At present, the exact mechanism of particle formation in the presence of Tween 80 and SDS (at different concentration levels) along with ultrasound is not clear (vis-à-vis the mechanisms reported in the literature $\mathrm{e}^{29-31}$ ). Ascertaining an exact mechanism will require a detailed analysis which is currently an ongoing research effort in our group.

The hypothesized mechanism of particle formation in the presence of large polymeric molecules along with ultrasound, however, can be proposed based on the possible interactions between curcumin molecules and the stabilizer molecules. The curcumin particles precipitated with ultrasound and BSA as a stabilizer are spherical and are made by aggregation of several spherical and cubic curcumin nanoparticles. The zeta potential of these particles was found to be $-42 \mathrm{mV}$, indicating favourable interaction between BSA and curcumin molecules. BSA at pH 6.5 (all the experiments in this work had a suspension $\mathrm{pH}$ in the range of 6-7) contains only deprotonated hydroxyl groups $(-\mathrm{COOH})$ on the surface, whereas $-\mathrm{NH}_{2}$ groups are embedded within the globular structure of BSA. ${ }^{32}$ Therefore, BSA can interact at the ends of the curcumin molecule at phenolic - $\mathrm{OH}$ and methoxyl groups resulting in a quick arrest of curcumin particle growth in all directions (schematic shown in Fig. 10). This may result in spherical primary nanoparticles precipitated in the presence of BSA. These primary units agglomerate further to form spherical superparticles.

HPMC, which is a neutral polymer, has several groups (such as $-\mathrm{OH}$ and $-\mathrm{OCH}_{3}$ ) which are capable of hydrogen bonding with curcumin molecules. However, a curcumin molecule has only one deprotonized group in the $\mathrm{pH}$ range 


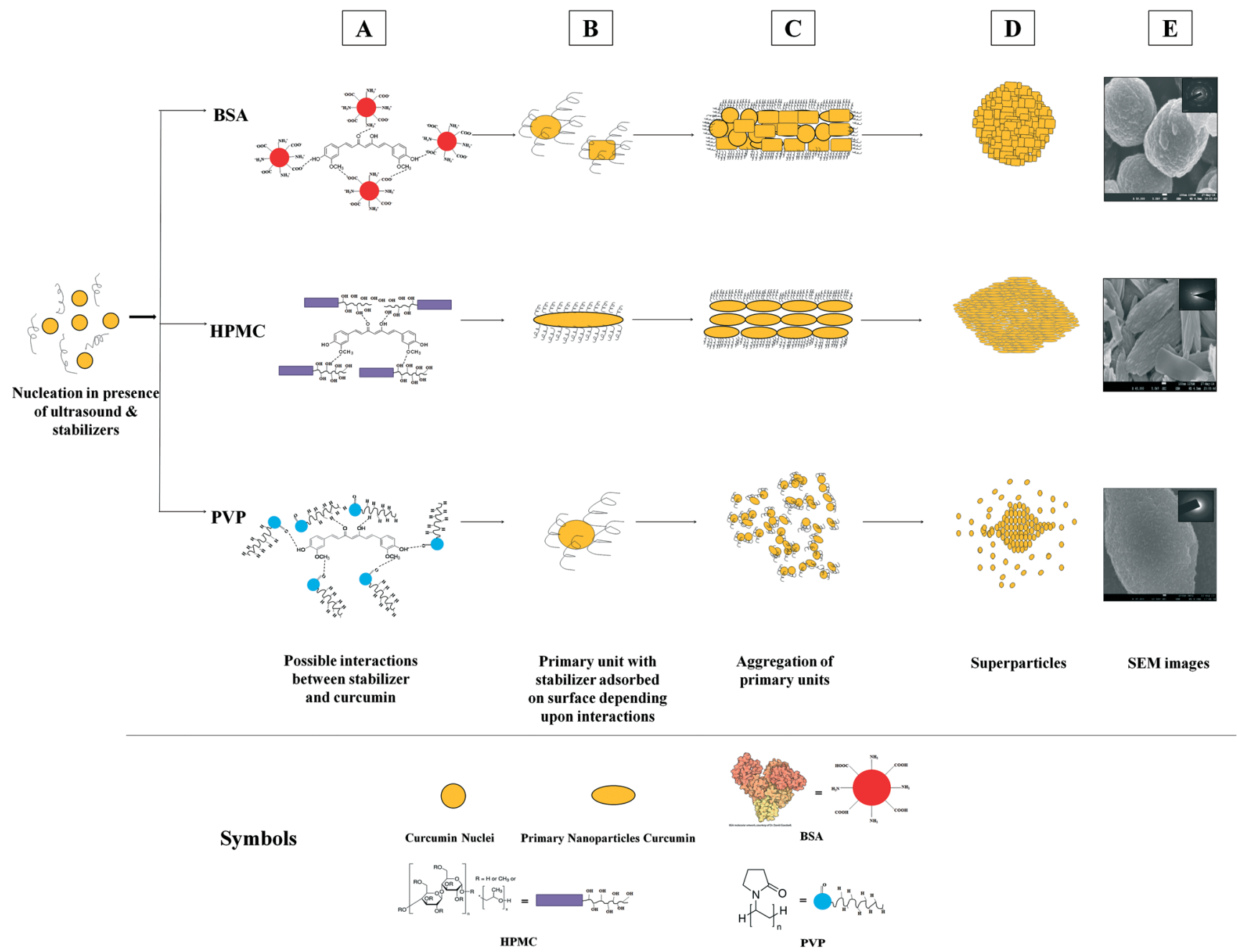

Fig. 10 Hypothesized mechanism for formation of curcumin superparticles in the presence of ultrasound and with large-molecule stabilizers. (A) The possible interactions between primary curcumin particle and stabilizers. (B) Morphology of a primary unit formation depending on the specific interactions between stabilizers and curcumin. (C) Aggregation of the primary unit during particle formation. (D) Final superstructure formed by arrangements of primary units depending upon the stabilizers. (E) SEM image of curcumin particles formed with different stabilizers and their SAED patterns (inset).

of $6-12,{ }^{33}$ i.e. enolic $-\mathrm{OH}$, which can possibly be involved in hydrogen bonding (all the experiments in this work had a suspension $\mathrm{pH}$ in the range of 6-7). The functional groups of $-\mathrm{OH}$ or $-\mathrm{OCH}_{3}$ from $\mathrm{HPMC}$ can interact through hydrogen bonding with the deprotonated enolic $-\mathrm{OH}$ of curcumin which is on the lateral side of a curcumin molecule. This could further be confirmed by the zeta potential (ZP) of curcumin particles precipitated with HPMC. The ZP of these particles was found to be $-5 \mathrm{mV}$, whereas the $\mathrm{ZP}$ of bare curcumin particles precipitated with ultrasound and without HPMC was found to be $-38 \mathrm{mV}$. HPMC being neutral, its interaction with curcumin molecules at the enolic $-\mathrm{OH}$ position masks the negative charge and reduces the negative value of the $\mathrm{ZP}$ of curcumin particles. The small rod-like primary units of curcumin particles are formed (as evident from the SEM micrographs shown in Fig. 2k and l) due to the adsorption of HPMC on the lateral sides of the curcumin nanoparticles. These HPMC-coated rod-like primary nanoparticles would further aggregate (by end-to-end attachment) along the $c$ axis and form rice seed-like particles (schematic shown in Fig. 10).

In the case of PVP as a stabilizer, spherical primary nanoparticles are formed, which further aggregate to form spherical superparticles (Fig. 10). The zeta potential of particles precipitated with PVP and ultrasound was found to be $-27 \mathrm{mV}$. In this case, a spherical shape of primary nanoparticles could be a result of effective stabilization of curcumin particles through hydrogen bonding with curcumin molecules as evident from the FTIR analysis which was carried out to understand the possible interactions of stabilizers with curcumin (Fig. S18 $\dagger$ ). FTIR analysis reveals that major changes occur in the case of particles precipitated with ultrasound and PVP only in the frequency region corresponding to $-\mathrm{OH}$ interactions in comparison to other particles precipitated with other stabilizers. This observation indicates that PVP is a better stabilizer 
compared to other stabilizers. Such favorable interactions of PVP with curcumin molecules result in the formation of spherical and amorphous primary nanoparticles (also evident from XRD and SAED patterns), which aggregate further to form superparticles.

Thus, the formation of curcumin particles in the presence of ultrasound only (and no stabilizer) and in the presence of ultrasound along with polymeric stabilizers such as BSA, HPMC, and PVP seems to follow a non-classical crystallization pathway. Generally, a non-classical pathway of particle formation is followed when a large number of temporarily stabilized nanoparticles are formed and levels of supersaturation for particle growth decrease to a low value due to the consumption of supersaturation for large nucleation rates. ${ }^{13}$ The use of ultrasound and additives leads to large nucleation rates (due to enhanced diffusion coefficients of solute molecules $^{3,28}$ and reduction in surface tension at the solid-liquid interface $\left.{ }^{3}\right)$. These large nucleation rates coupled with integration-controlled growth of particles $^{31}$ result in a large population of nanoparticles and a reduced level of supersaturation. In addition to this, ultrasound and stabilizers also facilitate stabilization of nanoparticles by converting thermodynamically unstable matter into a more stable form ${ }^{5,28}$ and by preventing the growth of the particles due to adsorbed stabilizer molecules, ${ }^{26,34}$ respectively. These primary stabilized nanoparticles then aggregate randomly in the presence of stabilizers and ultrasound to form secondary superparticles. The shape, morphology and crystallinity of these superstructures depend upon the interactions of stabilizers with curcumin molecules. The particles precipitated in the presence of largemolecule stabilizers such as HPMC, BSA and PVP seem to undergo slower crystallographic fusion mainly due to occlusion of large polymeric molecules in the superstructure. The presence of these polymeric stabilizers prevents reorientation and fusion of primary nanoparticles resulting in the formation of superstructures. ${ }^{35,36}$ As opposed to this, the particles precipitated in the presence of small-molecule stabilizers such as Tween 80 and SDS along with ultrasound looked more fused. Additional research is needed to ascertain an exact particle formation mechanism in these two cases.

\section{Conclusions}

Precipitation of a poorly water-soluble drug, curcumin, has been carried out from its ethanolic solutions using water as an antisolvent in the presence of ultrasound and stabilizers. SEM analysis of precipitated particles reveals an interesting morphological behavior of curcumin particles when precipitated in the presence of ultrasound and stabilizers. The absence of ultrasound during precipitation results in dendritic particles due to a diffusion-limited growth in the absence of uniform mixing, whereas the use of ultrasound alone results in a rice seed-like particle morphology. Precipitation of curcumin particles in the presence of ultrasound and (with or without) stabilizers seems to follow a nonclassical crystallization pathway. The use of ultrasound and stabilizers leads to large nucleation rates and hence to a large population of stabilized nanoparticles. These stabilized nanoparticles assemble under the influence of ultrasound to form superstructures. Curcumin particles precipitated in the presence of ultrasound and with SDS as well as Tween 80 have a rice seed-like morphology and appear to be quite fused. On the other hand, curcumin particles precipitated with polymeric stabilizers such as BSA, HPMC, and PVP clearly look like the aggregates formed via assembly of several nanoparticles. The particles precipitated in the presence of BSA and ultrasound appear to be spherical aggregates $\sim 1 \mu \mathrm{m}$ in size made by aggregation of several primary nanoparticles. The primary constituents of these superparticles were found to be plate-like as well as spherical nanoparticles 100-200 $\mathrm{nm}$ in size. In the presence of ultrasound and HPMC the rice seed-like superparticles of $\sim 2-5 \mu \mathrm{m}$ were obtained when precipitation of curcumin was carried out. The primary constituents of these particles were found to be small rod-shaped nanoparticles around $100 \mathrm{~nm}$ in size. Precipitation of curcumin in the presence of PVP and ultrasound results in spherical pearl-like aggregates $\sim 1.5 \mu \mathrm{m}$ in size, where the primary units were spherical nanoparticles with a size of $\sim 50-80 \mathrm{~nm}$. Thus, it can be proposed that the polymeric stabilizers prevent crystallographic fusion and hence the particles appear as loose aggregates, whereas smallmolecule stabilizers like SDS and Tween 80 may not be able to prevent fusion of individual particles and hence these particles look more fused. It is also possible that the process of particle growth via molecule-by-molecule addition may have been followed. Further research is needed to ascertain an exact mechanism of particle formation in these cases.

Ultrasound induces formation of different polymorphs of curcumin as compared to the starting material. While raw curcumin particles are in the monoclinic form, the particles precipitated in the presence of ultrasound and stabilizers mainly conform to the orthorhombic form of curcumin. The polymorphic transformations are induced mainly due to prevention of hydrogen bonding among curcumin molecules (which is responsible for macrocyclic ring formation in the monoclinic form) by ultrasound.

The results presented in this work demonstrate that the particle formation pathways of curcumin could be manipulated to obtain desired morphological and polymorphic characteristics through a judicious choice of stabilizers and mixing techniques (ultrasound vs. stirring). Moreover, this methodology could be applied to other poorly water-soluble drugs as well as other organic and inorganic materials which can be precipitated by the liquid antisolvent technique.

\section{Acknowledgements}

We gratefully acknowledge financial support from the Department of Science and Technology (DST), Government of India, and the Indian Institute of Technology, Gandhinagar (IITGN). We would like to thank Dr. Abhijit Mishra (IITGN) and Dr. Palash Sanphui (IISC Bangalore) for discussions regarding 
PXRD analysis. We are also thankful to Dr. Emila Panda (IITGN) for discussions on HR-TEM analysis. Further, we would also like to thank the ICT-Mumbai, the NIPER-Mohali and the CSMCRIBhavnagar for providing access to their HR-TEM facility.

\section{Notes and references}

1 A. A. Thorat and S. V. Dalvi, Chem. Eng. J., 2012, 181-182, $1-34$.

2 E. Merisko-Liversidge, G. G. Liversidge and E. R. Cooper, Eur. J. Pharm. Sci., 2003, 18, 113-120.

3 S. V. Dalvi and R. N. Dave, Int. J. Pharm., 2010, 387, 172-179.

4 Y. He, Y. Huang and Y. Cheng, Cryst. Growth Des., 2010, 10, 1021-1024.

5 D. Xia, M. Ouyang, J. X. Wu, Y. Jiang, H. Piao, S. Sun, L. Zheng, J. Rantanen, F. Cui and M. Yang, Pharm. Res., 2012, 29, 158-169.

6 Y. Ma, H. Cölfen and M. Antonietti, J. Phys. Chem. B, 2006, 110, 10822-10828.

7 Y. Ma, H. G. Börner, J. Hartmann and H. Cölfen, Chem. Eur. J., 2006, 12(30), 7882-7888.

8 D. D. Medina and Y. Mastai, Cryst. Growth Des., 2008, 8, 3646-3651.

9 F. Benaouda, Z. Bachoo, M. B. Brown, G. P. Martin and S. A. Jones, Soft Matter, 2013, 9, 10165-10173.

10 T. Lee and C. Zhang, Pharm. Res., 2008, 25, 1563-1571.

11 Y. Jiang, L. Gower, D. Volkmer and H. Cölfen, Cryst. Growth Des., 2011, 11, 3243-3249.

12 M. Ejgenberg and Y. Mastai, Cryst. Growth Des., 2012, 12, 4995-5001.

13 H. Cölfen and M. Antonietti, Mesocrystal Systems, in Mesocrystals and Nonclassical Crystallization, John Wiley \& Sons, Ltd, 2008, pp. 113-177.

14 Y. Su, X. Yan, A. Wang, J. Fei, Y. Cui, Q. Heab and J. Li, J. Mater. Chem., 2010, 20, 6734-6740.

15 B. B. Aggarwal and K. B. Harikumar, Int. J. Biochem. Cell Biol., 2009, 41, 40-59.

16 P. Sanphui, N. R. Goud, U. B. R. Khandavilli, S. Bhanoth and A. Nangia, Chem. Commun., 2011, 47, 5013-5015.
17 M. K. Mishra, P. Sanphui, U. Ramamurty and G. R. Desiraju, Cryst. Growth Des., 2014, 14, 3054-3061.

18 D. Xia, P. Quan, H. Piao, S. Sun, Y. Yin and F. Cui, Eur. J. Pharm. Sci., 2010, 40, 325-334.

19 M. Louhi-Kultanen, M. Karjalainen, J. Rantanen, M. Huhtanen and J. Kallas, Int. J. Pharm., 2006, 320, 23-29.

20 M. Kurotani and I. Hirasawa, J. Cryst. Growth, 2008, 310, 4576-4580.

21 H. Hatakka, H. Alatalo, M. Louhi-Kultanen, I. Lassila and E. Haeggstrom, Chem. Eng. Technol., 2010, 33, 751-756.

22 Y. Gong, B. M. Collman, S. M. Mehrens, E. Lu, J. M. Miller, A. Blackburn and D. J. W. Grant, J. Pharm. Sci., 2008, 97, 2130-2144.

23 Y. Zhu, Y. Liu, Q. Ruan, Y. Zeng, J. Xiao, Z. Liu, L. Cheng, F. Xu and L. Zhang, J. Phys. Chem. C, 2009, 113, 6584-6588.

24 H. Uchiyama and H. Imai, Langmuir, 2008, 24, 9038-9042.

25 O. D. Linnikov, Desalination, 1999, 122, 1-13.

26 S. V. Dalvi and R. N. Dave, Ind. Eng. Chem. Res., 2009, 48, 7581-7593.

27 C. Beck, S. V. Dalvi and R. N. Dave, Chem. Eng. Sci., 2010, 65, 5669-5675.

28 A. A. Thorat, M. D. Yadav and S. V. Dalvi, Langmuir, 2014, 30, 4576-4592.

29 M. Bujan, M. Sikirić, N. Filipović-Vinceković, N. Vdović, N. Garti and H. Füredi-Milhofer, Langmuir, 2001, 17, 6461-6470.

30 N. Rodríguez-Hornedo and D. Murphy, J. Pharm. Sci., 2004, 93, 449-460.

31 G. A. Ilevbare, H. Liu, K. J. Edgar and L. S. Taylor, Cryst. Growth Des., 2012, 12, 6050-6060.

32 P. Nithiyasi, K. Balaji, P. Brindha and M. Parthasarathy, Nanotechnology, 2012, 23, 8.

33 Z. Wang, M. H. M. Leung, T. W. Kee and D. S. English, Langmuir, 2010, 26, 5520-5526.

34 S. L. Raghavan, A. Trividic, A. F. Davis and J. Hadgraft, Int. J. Pharm., 2001, 212, 213-221.

35 A. N. Kulak, P. Iddon, Y. Li, S. P. Armes, H. Cölfen, O. Paris, R. M. Wilson and F. C. Meldrum, J. Am. Chem. Soc., 2007, 129, 3729-3736.

36 L. Zhou and P. O'Brien, Small, 2008, 4, 1566-1574. 